\title{
Campylobacteriosis in returning travellers and potential secondary transmission of exotic strains
}

\author{
L. MUGHINI-GRAS ${ }^{1,2,3}$, J. H. SMID ${ }^{2,6}$, J.A. WAGENAAR ${ }^{3,4,5}$, A. DE BOER ${ }^{4}$, \\ A. H. HAVELAAR ${ }^{2,6}$, I. H. M. FRIESEMA ${ }^{2}$, N.P. FRENCH ${ }^{7}$, C. GRAZIANI ${ }^{1}$, \\ L. BUSANI ${ }^{1}$ AND W. VAN PELT ${ }^{2}$ \\ ${ }^{1}$ Department of Veterinary Public Health and Food Safety, Istituto Superiore di Sanità, Rome, Italy \\ ${ }^{2}$ Centre for Infectious Disease Control, National Institute of Public Health and the Environment (RIVM), \\ Bilthoven, The Netherlands \\ ${ }^{3}$ Department of Infectious Diseases and Immunology, Utrecht University, Utrecht, The Netherlands \\ ${ }^{4}$ Central Veterinary Institute of Wageningen UR, Lelystad, The Netherlands \\ ${ }^{5}$ WHO Collaborating Centre for Reference and Research on Campylobacter/OIE Reference Laboratory \\ for Campylobacteriosis, Utrecht/Lelystad, The Netherlands \\ ${ }^{6}$ Institute for Risk Assessment Sciences, Utrecht University, Utrecht, The Netherlands \\ ${ }^{7}$ Massey University, Palmerston North, New Zealand
}

Received 19 March 2013; Final revision 4 July 2013; Accepted 31 July 2013;

first published online 21 August 2013

\section{SUMMARY}

Multilocus sequence types (STs) were determined for 232 and 737 Campylobacter jejunilcoli isolates from Dutch travellers and domestically acquired cases, respectively. Putative risk factors for travel-related campylobacteriosis, and for domestically acquired campylobacteriosis caused by exotic STs (putatively carried by returning travellers), were investigated. Travelling to Asia, Africa, Latin America and the Caribbean, and Southern Europe significantly increased the risk of acquiring campylobacteriosis compared to travelling within Western Europe. Besides eating chicken, using antacids, and having chronic enteropathies, we identified eating vegetable salad outside Europe, drinking bottled water in high-risk destinations, and handling/eating undercooked pork as possible risk factors for travel-related campylobacteriosis. Factors associated with domestically acquired campylobacteriosis caused by exotic STs involved predominantly person-to-person contacts around popular holiday periods. We concluded that putative determinants of travel-related campylobacteriosis differ from those of domestically acquired infections and that returning travellers may carry several exotic strains that might subsequently spread to domestic populations even through limited person-to-person transmission.

Key words: Multilocus sequence typing, person-to-person transmission, risk factor, source attribution, travel-related campylobacteriosis.

\section{INTRODUCTION}

Diarrhoeal infections remain a major concern for travellers, especially for those bound for destinations

\footnotetext{
* Author for correspondence: Dr L. Mughini-Gras, Rijksinstituut voor Volksgezondheid en Milieu (RIVM), Centre for Infectious Disease Control $(\mathrm{CIb})$, Epidemiology and Surveillance Unit, PO Box 1, 3720 BA Bilthoven, The Netherlands.

(Email: lapo.mughini.gras@rivm.nl or lapomugras@libero.it)
}

where relatively substandard hygienic conditions exist. A Dutch study showed that in a sample of 1202 individuals travelling to developing countries, $50 \%$ experienced one or more diarrhoeal episodes [1].

Campylobacteriosis is a leading cause of traveller's diarrhoea, particularly in travellers returning from Southern and South East Asia [2, 3]. In The Netherlands (16.5 million population), an estimated 
90000 cases of campylobacteriosis occur annually, with $\sim 12 \%$ estimated as international travel-related [4]. Moreover, fluoroquinolone-resistant Campylobacter infections occur significantly more often in travelrelated cases $[3,5]$.

Travellers are particularly prone to experiencing (symptomatic) Campylobacter infections [2, 6, 7], and susceptibility to disease is associated with duration of foreign stay. For instance, duration of residence of expatriates in Nepal was linearly correlated with protection from diarrhoeal infection [8], and travellers experiencing multiple diarrhoeal episodes had a shorter duration of symptoms after the first episode [1]. Similarly, in expatriates in Thailand, campylobacteriosis occurred significantly more often in those living there for $<1$ year [9]. A documented case of acquired immunity in developed countries concerned people professionally exposed. Newly employed poultry abattoir workers in Sweden have been shown to suffer more often from campylobacteriosis than their longer employed colleagues [10]. If partial immunity to (severe) disease is acquired over time with repeated exposure, then such protection should correlate with age. Indeed, campylobacteriosis incidence peaks in infancy worldwide and older age groups are significantly less prone to infection with common Campylobacter strains compared to the young [11]. Moreover, Swedish travellers to countries such as Germany, France, Belgium, The Netherlands, Austria, Luxembourg or Switzerland, all of which are developed countries with high hygienic standards, still have a 4.4-21 times higher risk of acquiring campylobacteriosis compared to those travelling to neighbouring Norway [12]. A recent study comparing Campylobacter multilocus sequence typing (MLST) datasets from different countries has further highlighted the importance of geographical distance in strain dissimilarity [13]. Moreover, serological studies of patients and volunteers infected with campylobacters (reviewed in [14]) have revealed an array of immunogenic components and elicited antibodies displayed little cross-reactivity, indicating considerable antigenic variation. Although core-genome (as described by MLST) is not necessarily related to antigens, and cross-protection is expected to develop for strains sharing similar antigenic properties, the higher risk of campylobacteriosis in travellers does not appear to be limited just to higher levels of exposure in developing countries, but also to the possible presence of 'new' Campylobacter strains, endemic in the different regions to which travellers have rarely been exposed before [14]. It follows that, probabilistically, these new strains are more likely to be associated with regionally untested antigens than widespread strains, and acquired protection may be ineffective when exposed to uncommon strains, as evidenced by a Canadian study [15].

It is conceivable that the infected, but not necessarily symptomatic [14], returning travellers may introduce into the domestic population several 'exotic' strains with a higher probability of possessing antigens that are underrepresented in the local reservoirs, i.e. food-producing animals, pets and wildlife. These exotic strains may therefore constitute a distinctive, primarily human-restricted Campylobacter population that may have at least the potential to spread domestically via the person-to-person route. Although Campylobacter person-to-person transmission is believed to be uncommon and up to $66 \%$ and $21 \%$ of laboratory-confirmed cases in The Netherlands are attributable to chicken and cattle, respectively [16], it has been shown that campylobacteriosis household outbreaks are more common than believed [17] and up to $18 \%$ of putatively household outbreak-related cases are suggestive of secondary spread [18]. This raises the question to what extent exotically introduced strains may spill over into the domestic population and at first spread anthroponotically.

In this study, we investigated the MLST profiles of Campylobacter strains isolated from travellers returning to The Netherlands compared to those from domestically acquired cases. We also investigated putative determinants of travel-related campylobacteriosis by comparing the exposures of the returned travellers with those of travellers in the control population. Furthermore, we used a population genetics model for source attribution to estimate the probability that the domestically acquired infections were caused by exotic strains, putatively carried by returned travellers. Finally, factors associated with exotically introduced domestic campylobacteriosis were investigated and the percentage of domestically acquired cases due to person-to-person transmission of exotic strains was estimated.

\section{METHODS}

\section{Data}

An earlier case-control study on risk factors for campylobacteriosis conducted in The Netherlands between April 2002 and March 2003, the 'CaSa study' [6], formed the basis of this study. Isolates of 
3115 Campylobacter jejunilcoli cases identified by the Dutch Regional Public Health Laboratories (RPHL) through passive surveillance were sent to the Dutch Central Veterinary Institute (CVI) for molecular speciation $[19,20]$. Controls were selected from RPHL population registries by frequency matching (aiming at two per case) according to age $(0-4,5-17,18-29$, $30-44,45-59, \geqslant 60$ years), sex, urbanization degree (urban: $>2500$ addresses $/ \mathrm{km}^{2}$; urbanized: 500-2500 addresses $/ \mathrm{km}^{2}$; rural: $<500$ addresses $/ \mathrm{km}^{2}$ ) and season (April-June 2002, July-September 2002, OctoberDecember 2002, January-April 2003) [6, 16, 21]. Cases and controls were asked to complete a questionnaire regarding foreign travel, food consumption, kitchen hygiene, contact with animals, contact with gastroenteritis cases, occupation, recreational activities, medication use and chronic disease history. Questions covered the 7 days prior to symptom onset (cases) or questionnaire completion (controls). Missing values were handled using multiple imputation $[6,21]$.

Cases/controls not returning the questionnaire and/ or living abroad were excluded, leaving 1428 cases and 3363 controls enrolled in the study. Of these, 328 cases and 244 controls had travelled abroad with $\geqslant 1$ overnight stay in the destination country. A total of 66 countries were visited, with 36 cases and 27 controls visiting $>1$ country during the same travel episode. For three cases and four controls the travel destination was unknown. Destination countries were grouped into travel regions by adapting the United Nations geoscheme (http://unstats.un.org/unsd/methods/m49/ m49regin.htm\#europe).

Isolates from 737 non-travellers (domestically acquired cases) and 232 travellers were typed using MLST [22]. Association of the travellers' five most frequent sequence types (STs) and clonal complexes (CCs) with travel regions was tested using $\chi^{2}$ or Fisher's exact tests. Proportional Similarity Index (PSI) [13] was used to measure the (dis)similarity between ST frequency distributions of travellers and non-travellers. The PSI ranges between 0 (no common ST) and 1 (identical distributions). Simpson's index of diversity was calculated to define the ST diversity of travellers and non-travellers as the probability that two randomly selected individuals were infected with different STs [23].

\section{Source attribution}

The Asymmetric Island (AI) model, a Bayesian population genetics algorithm for modelling Campylobacter evolution and transmission [24], was used to estimate the probability $(P r)$ of the 737 non-travellers being infected with exotically introduced STs or with STs originating from four putative animal reservoirs (chicken, cattle, sheep, pig) or from the environment (water, sand, wild birds), a proxy for other unidentified reservoirs putatively of wildlife origin [16]. This study was restricted to campylobacteriosis of probable exotic origin. Results regarding the other animal and environmental sources have been reported elsewhere [13, 16].

To run the AI model, $C$. jejunilcoli MLST data from the above-mentioned animal and environmental sources were supplied by the CVI and supplemented with other data $[22,25,26]$ to provide a representative dataset for each source (Table 1). Supplementary data were selected from other published datasets (reported in [13]) using Smid's methodology, which allows for the selection of non-local (and/or non-recent) MLST datasets for Campylobacter source attribution while minimizing potential biases $[13,16]$. Differences in $P r$ for exotic origin $\left(P r_{\mathrm{e}}\right)$ were tested for the variables age, sex, and season using Kruskal-Wallis or MannWhitney tests.

\section{Factors associated with travel-related campylobacteriosis}

Logistic regression was used to investigate putative risk factors for travel-related campylobacteriosis. The 325 diseased travellers and the 238 healthy travellers with known travel destination were included as cases and controls, respectively. Analysis was performed in the same way as in previous studies $[6,16,21]$. A total of 131 factors were tested for association with Campylobacter infection in the preliminary single-variable analysis and those factors showing $P \leqslant 0 \cdot 10$ were selected for inclusion in a multivariable model. A backward stepwise procedure was applied and variables with $P<0.05$ were retained in the final model. Education level [16], travel region, and length of stay (days) were always included as covariates to control for confounding in addition to the frequencymatched variables. As travel regions were almost mutually exclusive, Western Europe (which The Netherlands belongs to) was made the base category against which the other regions were assessed.

To explore if the risk factors of the reduced model differed according to age, sex, education level, season, and travel region, we also tested for their two-way interactions. The final multivariable model was then 
Table 1. Campylobacter jejuni/coli strains typed with MLST used to feed the Asymmetric Island model for source attribution

\begin{tabular}{llcccccl}
\hline \hline Country & $\begin{array}{l}\text { Humans } \\
\text { (travellers) }\end{array}$ & Chicken & Cattle & Sheep & Pig & Environment & Ref. \\
\hline The Netherlands & $232^{\mathrm{a}}$ & 236 & 0 & 9 & 0 & 106 (water) & Data $^{\mathrm{b}}$ \\
United Kingdom & 0 & 73 & 46 & 46 & 72 & 50 (sand) & {$[22]$} \\
Scotland & 0 & 239 & 90 & 90 & 88 & 133 (wild birds) & {$[25]$} \\
Switzerland & 0 & 77 & 23 & 23 & 0 & 0 & {$[26]$} \\
Total & 232 & 625 & 168 & 168 & 160 & 289 & \\
\hline \hline
\end{tabular}

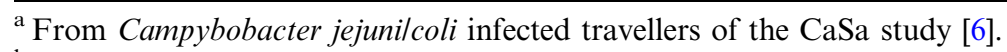

${ }^{\mathrm{b}}$ Provided by the Central Veterinary Institute (CVI) in Lelystad, The Netherlands.

expanded to include significant interactions. Overall model significance and goodness-of-fit were verified by likelihood ratio $\chi^{2}$ and Hosmer-Lemeshow tests, respectively. The best-fitting model was identified using Akaike's Information Criterion (AIC). Biascorrected bootstrap confidence intervals were also calculated (1000 iterations) and compared to the standard ones. As these did not differ significantly, the standard values were reported. Statistical analysis was performed using Stata v. 11.2 (StataCorp., USA).

\section{Factors associated with exotically introduced domestic campylobacteriosis}

To investigate putative risk factors for domestically acquired campylobacteriosis caused by STs of probable exotic origin, MLST data of travellers were included as an additional source in the AI model. Similar to previous studies [16, 27, 28], the $P r_{\mathrm{e}}$ distribution was assessed and a cut-off was determined to optimize the number of domestic cases assigned to be exotically introduced and the confidence as to their correct assignment derived by the highest possible $P r_{\mathrm{e}}$. Logistic regression was then used to investigate risk factors for domestically acquired campylobacteriosis caused by STs with at least $77 \%$ probability (cut-off $P r_{\mathrm{e}} \geqslant 0 \cdot 77$ ) of originating from abroad. This cut-off $P r_{\mathrm{e}}$ resulted in the selection of 79 cases with a median $P r_{\mathrm{e}}$ of 0.89 (mean 0.88 , range 0.77-0.99) belonging to 35 different STs. The 3119 non-travelling controls were included in this analysis.

The effect of the assignment cut-off $P r_{\mathrm{e}}$ on the risk factors was checked by sensitivity analysis, which was done by repeating the analysis for different cut-off $P r_{\mathrm{e}}$ values from $0 \cdot 5$ to $0 \cdot 9$. Low numbers of cases did not allow for the construction of models based on a cut-off of $P r_{\mathrm{e}}>0.9$. Finally, a case-case analysis comparing exposures of domestic infections with exotic vs. non-exotic STs was performed.

\section{Estimation of domestic cases due to person-to-person transmission of exotic strains}

The percentage of domestic cases due to personto-person transmission of exotic strains, denoted by $\gamma$, was estimated as:

$\gamma=\frac{\left[1-\prod_{i=1}^{n}\left(1-\mathrm{PAR}_{i}\right)\right] \times N_{\mathrm{ED}}}{N_{\mathrm{D}}} \times 100$,

where PAR is the population attributable risk (calculated based on multivariable odds ratios and prevalence of exposure in cases) for factor $i$ out of $n$ factors entailing person-to-person transmission in the final multivariable model. $N_{\mathrm{ED}}$ is the estimated total number of domestic cases of probable exotic origin, given by $\operatorname{Binomial}\left(N_{\mathrm{D}}, \operatorname{Pr}\right)$, where $N_{\mathrm{D}}$ is the total number of domestic cases (non-travellers) enrolled in the study and $P r$ is the estimated probability for a domestic case to be infected with an exotic ST, assumed to be $\operatorname{Beta}(a, b)$-distributed, with $a$ being the 79 typed domestic cases with $P r_{\mathrm{e}} \geqslant 0.77$ and $b$ being the total number of typed domestic cases $(n=737)$ minus 79. Estimation was performed using @RISK (Palisade Corp., USA) by setting 10000 iterations with the Latin Hypercube sampling technique and a seed of 1 .

\section{RESULTS}

\section{Genotypes}

The 737 typed strains from non-travellers were assigned to $154 \mathrm{STs}$ and $28 \mathrm{CCs}$, whereas those from the 232 travellers were assigned to 127 STs and 23 CCs. Twenty-eight STs from non-travellers and 23 STs from travellers were unassigned to previously identified CCs. 
(a)

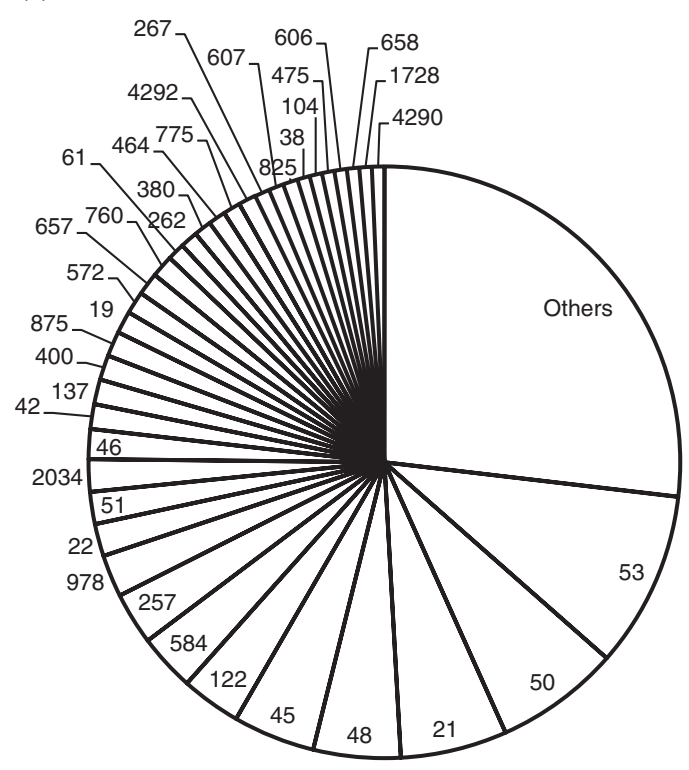

(b)

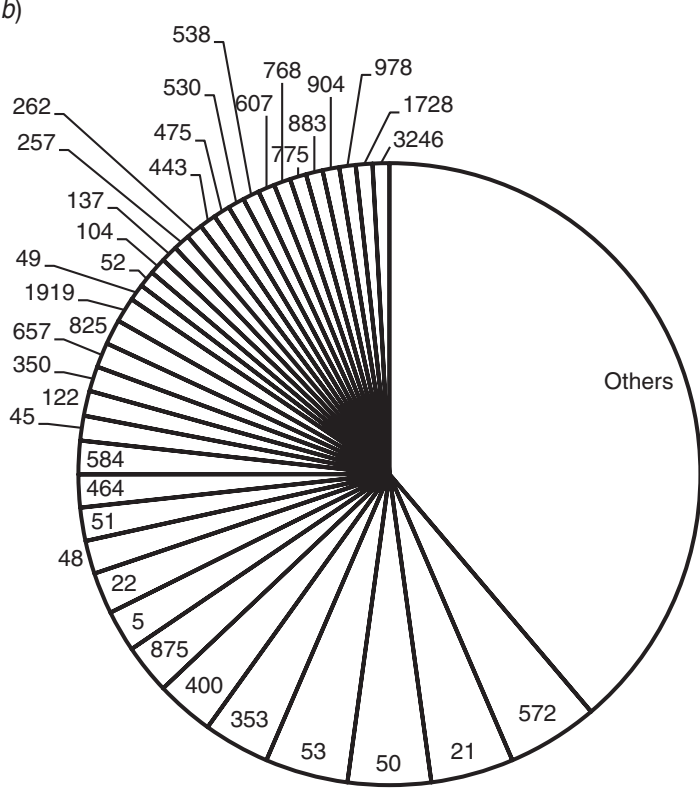

Fig. 1. Sequence types identified in Campylobacter jejunilcoli isolates from (a) 737 non-travellers (infections acquired in The Netherlands) and (b) 232 travellers returning to The Netherlands. Category 'others' includes sequence types that occurred less than five times (non-travellers) and less than twice (travellers).

In non-travellers, the top five STs (ST53, ST50, ST21, ST48, ST45) accounted for $>25 \%$ of cases and the top five CCs (CC21, CC45, CC206, CC257, CC48) for $>50 \%$ of cases (Fig. 1). In travellers, the top five STs (ST572, ST21, ST50, ST53, ST353) accounted for $\sim 20 \%$ of cases and the top five CCs (CC21, CC353, CC828, CC206, CC52) for $>50 \%$ of cases (Fig. 2). STs occurring once accounted for $46 \%$ and $70 \%$ of STs in non-travellers and travellers, respectively. PSI between travellers and non-travellers was 0.47 [95\% confidence interval (CI) $0.34-0.59]$ while Simpson's index was 0.972 (95\% CI 0.968-0.976) in non-travellers and 0.988 (95\% CI $0.984-0.992)$ in travellers.

There were 68 STs ( 74 cases) found only in travellers and absent in any of the considered sources and in non-travellers (Table 2). Most (73\%) cases infected with these traveller-only STs had travelled to Asia or Africa compared to $46 \%$ of all travel-related cases returning from these continents ( $z$ test, $P<0.001)$. Conversely, $23 \%$ of cases infected with traveller-only STs had travelled within Europe compared to 53\% of all travel-related cases travelling within Europe ( $z$ test, $P<0 \cdot 001$ ).

ST572 was significantly overrepresented $(64 \%)$ in travellers from Western Europe $(P=0.001)$; ST50 in those from Western Asia $(40 \%, P=0.014)$; ST53 in those from Southern Europe $(50 \%, P=0.035)$; ST353, CC353 and CC828 in those from Northern
Africa $(38 \%, 27 \%$, and $46 \% ; P=0.003, P<0.001$, and $P<0.001$, respectively); CC52 in those from Eastern Europe $(23 \%, P=0.001)$.

STs with $P r_{\mathrm{e}} \geqslant 0.77$ included in the risk factor analysis for exotically introduced domestic campylobacteriosis were ST1009, ST400, ST353, ST4307, ST5, ST2100, ST4308, ST2088, ST2151, ST1728, ST2844, ST508, ST403, ST443, ST350, ST3130, ST2156, ST878, ST657, ST4269, ST2882, ST2130, ST1080, ST4300, ST2187, ST586, ST696, ST587, ST2641, ST2553, ST3015, ST2123, ST4311, ST4278 and ST4284. There was a significant seasonal effect $(P=0.036)$ on $P r_{\mathrm{e}}$, which peaked in October-December and decreased in April-June. No significant age and sex effects on $P r_{\mathrm{e}}$ were found.

\section{Factors associated with travel-related campylobacteriosis}

Compared to travelling within Western Europe, travelling to any region in Asia, Africa, Latin America and the Caribbean, and Southern Europe posed a higher risk of acquiring campylobacteriosis (Table 3), whereas the risk posed by Northern and Eastern Europe and Oceania, as well as the length of stay, were not significant $(P>0 \cdot 05)$.

Factors positively associated with travel-related campylobacteriosis (Table 3) were: using protonpump inhibitors, consuming vegetable salad when 
(a)

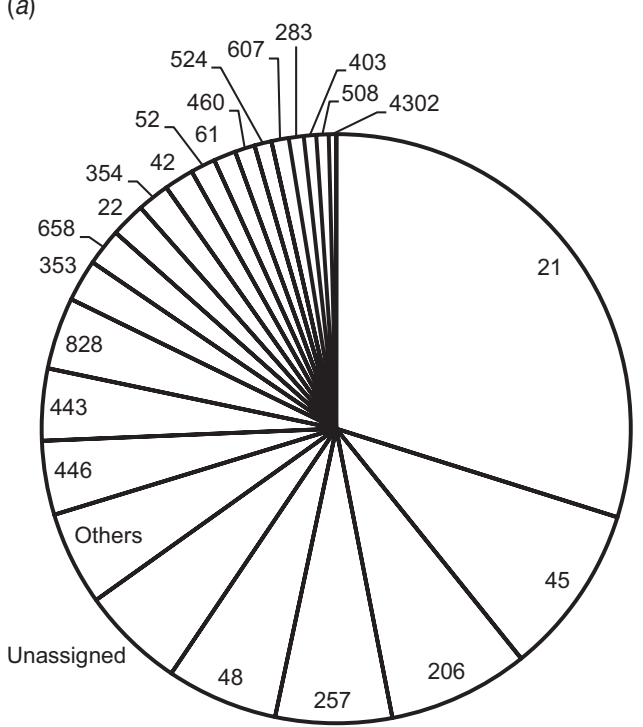

(b)

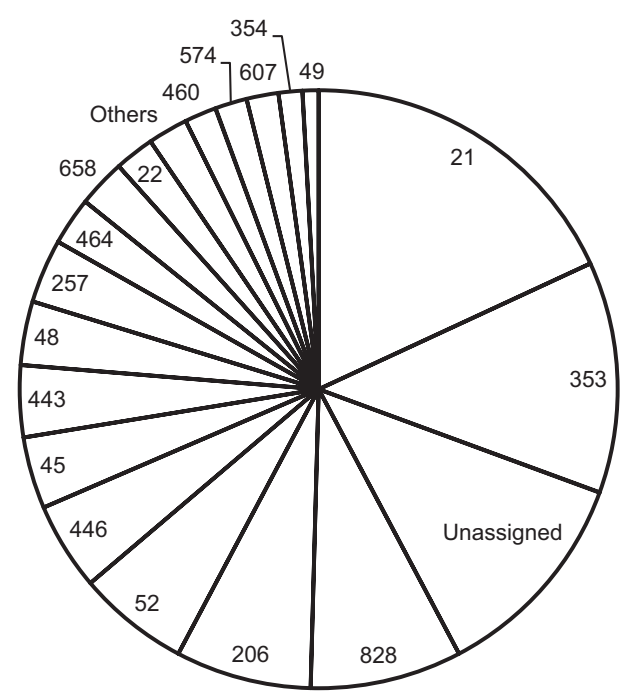

Fig. 2. Clonal complexes identified in Campylobacter jejuni/coli isolates from (a) 737 non-travellers (infections acquired in The Netherlands) and (b) 232 travellers returning to The Netherlands. Category 'others' includes clonal complexes that occurred less than twice (non-travellers) and less than once (travellers).

travelling outside Europe, handling and/or eating raw or undercooked pork, having chronic enteropathies, drinking bottled water when travelling to Southern Europe or non-European countries, and consuming chicken. Consuming yoghurt and being employed in healthcare were negatively associated with the outcome.

\section{Factors associated with exotically introduced domestic campylobacteriosis}

Factors positively associated with exotically introduced domestic campylobacteriosis (Table 4) were: not washing hands after toilet visit in January-April 2003, using proton-pump inhibitors, being a school-attending child in April-June 2002 and October-December 2002, attending public swimming pools in October-December 2002, and contact with gastroenteritis cases outside the household. Consuming yoghurt was negatively associated with the outcome. Sensitivity analysis of the cut-off $P r_{\mathrm{e}}$ revealed that this had no effect on the main results. In case-case analysis, the same factors were identified apart from consuming yoghurt and antacids (no longer significant), and therefore no further results are presented.

It was estimated that, on average, $3 \cdot 2 \%(95 \% \mathrm{CI}$ $2 \cdot 4-4 \cdot 1)$ of all domestic cases are due to personto-person transmission of exotically introduced STs.

\section{DISCUSSION}

The risk for Dutch travellers acquiring campylobacteriosis depended on the travel destination. Consistent with previous studies [2, 3], Asia, Africa, Latin America and the Caribbean, and Southern Europe were regions associated with an increased risk for campylobacteriosis compared to Western Europe, which comprises the neighbouring countries of The Netherlands. Regional risk variations may be due to differences in local epidemiology and hygiene standards. However, a cautious interpretation of these figures is warranted, as physicians may be more likely to decide on laboratory investigation when a gastroenteritis patient has travelled to a high-risk destination [2]. Although it is also possible for regional risk variation to be substantial in domestic travel [29], this may not apply to a relevant extent to The Netherlands, which is a relatively small country and is highly interconnected by high-speed routes and public transport, allowing for easy daily commute and travel, even from and to neighbouring countries such as Belgium and Germany, with very limited inbound overnight stay by the Dutch.

STs of travellers and non-travellers were relatively similar and travellers showed more ST diversity than non-travellers, a possible reflection of the numerous countries where they acquired infection. Moreover, some STs were more likely than others to infect travellers visiting specific regions, and travellers infected 
Table 2. Sequence types found exclusively in travellers returning to The Netherlands but not in cases acquired in The Netherlands, nor in any of the sourced animal and environmental reservoirs

\begin{tabular}{|c|c|c|}
\hline Sequence type & $\begin{array}{l}\text { No. of } \\
\text { cases }\end{array}$ & $\begin{array}{l}\text { Travel } \\
\text { destination }\end{array}$ \\
\hline 4291 & 1 & Austria \\
\hline 474 & 1 & Belgium \\
\hline 4274 & 1 & Bolivia, Peru \\
\hline 3878,4252 & 2 & Czech Republic \\
\hline $830,931,2229,2968$ & 4 & Egypt \\
\hline 3606 & 1 & Ethiopia \\
\hline 892 & 1 & France \\
\hline 1044 & 1 & Germany \\
\hline $446,451,1042,4288$ & 4 & India \\
\hline $\begin{array}{l}530,161,2031,2109 \\
2131,2393,2941,4270 \\
4281,4287,4289,4296\end{array}$ & 13 & Indonesia \\
\hline 4298 & 1 & Italy \\
\hline 4305 & 1 & Jordan \\
\hline 3630 & 1 & Jordan, Iraq \\
\hline 1380 & 1 & Kenya \\
\hline 466 & 1 & Luxemburg, France \\
\hline 4293 & 1 & Malaysia \\
\hline 1039,4309 & 2 & Mali \\
\hline $2116,3575,4165,4299$ & 4 & Morocco \\
\hline 986 & 1 & Nepal, China \\
\hline 1233,4053 & 2 & Peru \\
\hline 2895 & 1 & Philippines \\
\hline 614 & 1 & Poland \\
\hline 4277 & 1 & Portugal \\
\hline 904 & 2 & Portugal, Spain \\
\hline 4275 & 1 & Singapore \\
\hline $148,1710,4294,4313,4408$ & 5 & Spain \\
\hline $\begin{array}{l}1919,768,407,1953,2083 \\
2315,4303\end{array}$ & 10 & Thailand \\
\hline $\begin{array}{l}3246,303,305,2066,2184 \\
2275,3142\end{array}$ & 8 & Turkey \\
\hline 919 & 1 & Vietnam, Malaysia \\
\hline
\end{tabular}

with STs that were undetected domestically had travelled predominantly to distant destinations in Asia and Africa, suggesting that differences in STs are related, to some extent, to the geographical distance of the travel region compared to The Netherlands, with STs from nearby European countries being generally more similar than those from farther destinations [13]. Another study [29] showed nearly identical C. jejuni ST distributions in two English populations, but clear differences were found compared to Finland, Australia and New Zealand. Yet, differentiation was not proportional to distance, with the English sample being more similar to the samples from Australia and New Zealand than to the Finnish one, suggesting that similarities in STs between countries mainly reflect similarities of sources of infection rather than geographical proximity per se.

Consistent with [30], travel-related isolates had a significantly higher ST diversity than domestic isolates, with a different spectrum of MLST genotypes representative of the different countries. The larger ST diversity in travellers combined with the association of some STs with specific destinations is also consistent with the presence of heterogeneously distributed clones that are endemic in the different regions but not so prevalent elsewhere in the world. Regionally endemic STs have been identified, e.g. in Australia [31], New Zealand [32] and Curaçao [33], and may emerge because of clonal expansion, niche adaptation, geographical isolation and host immune selection [34]. Although so far there has been no evidence of ST-specific immune responses, it can be hypothesized that the chance of being exposed to a ST with uncommon antigens is somewhat higher for STs that are rarely, rather than commonly, encountered. STs that are associated with strong regional clustering would therefore pose a higher risk to travellers because of limited, prior (repeated) exposure in addition to issues related to sanitation failure. The risk posed by uncommon STs is also substantiated by their age distribution [16, 35]. For instance, the three most common STs in non-travellers were mainly found in the young relative to other STs, decreasing steadily with age (data not shown). Conversely, rare STs ( $<5$ isolates) occurred independently of age. According to interpretation of similar findings [11], it is likely that antigenic properties associated with the common STs are frequently encountered throughout life; thus, the young would be more susceptible because they have encountered these STs less often. In contrast, rare STs, more probably related to uncommon antigens, would have rarely been encountered by all age groups.

The original CaSa study was designed to investigate factors associated with domestic campylobacteriosis and not purposely with travel-related Campylobacter infection [6]. However, most risk exposures apply to some extent world-wide, and while the behaviour of travellers (and hence their exposure to campylobacters) may be different from the domestic population, it has been reported that most STs found in travellers returning to Scotland are still attributable to chicken in a similar proportion to that of domestic cases [29]. Although this study did not have the power 
Table 3. Multivariable odds ratios (OR) and corresponding 95\% confidence intervals (CI) of risk factors for Campylobacter jejuni/coli infection in travellers returning to The Netherlands

\begin{tabular}{|c|c|c|c|}
\hline Risk factor ( $\%$ of imputed missing values) & $\begin{array}{l}\% \text { cases } \\
\text { exposed } \\
(n=325)\end{array}$ & $\begin{array}{l}\% \text { controls } \\
\text { exposed } \\
(n=238)\end{array}$ & OR $(95 \% \mathrm{CI})^{\mathrm{a}}$ \\
\hline Duration of stay ${ }^{\mathrm{b}}(3)$ & & & $1 \cdot 0(0 \cdot 9-1 \cdot 1)$ n.s. \\
\hline \multicolumn{4}{|l|}{ Region of destination $^{\mathrm{c}}$} \\
\hline Western Europe ${ }^{\mathrm{d}}$ & $22 \cdot 8$ & $60 \cdot 0$ & Reference \\
\hline Northern Europe ${ }^{\mathrm{e}}$ & $3 \cdot 4$ & $8 \cdot 8$ & $0 \cdot 8(0 \cdot 3-2 \cdot 2)$ n.s. \\
\hline Eastern Europe $^{f}$ & $4 \cdot 3$ & $5 \cdot 0$ & $1 \cdot 1(0 \cdot 3-2 \cdot 2)$ n.s. \\
\hline Southern Europe ${ }^{\mathrm{g}}$ & $22 \cdot 8$ & $24 \cdot 8$ & $1 \cdot 7(1 \cdot 0-3 \cdot 3)$ \\
\hline Northern Africa $^{\mathrm{h}}$ & $8 \cdot 6$ & $1 \cdot 3$ & $10 \cdot 6(2 \cdot 3-49 \cdot 0)$ \\
\hline Sub-Saharan Africa ${ }^{\mathrm{i}}$ & $4 \cdot 3$ & $0 \cdot 4$ & $25 \cdot 4(2 \cdot 7-310 \cdot 7)$ \\
\hline Western Asia $^{j}$ & $13 \cdot 8$ & $2 \cdot 1$ & $10 \cdot 6(2 \cdot 8-39 \cdot 9)$ \\
\hline South East Asia and China ${ }^{\mathrm{k}}$ & $16 \cdot 3$ & $0 \cdot 8$ & $27 \cdot 8(4 \cdot 5-170 \cdot 9)$ \\
\hline Southern Asia ${ }^{1}$ & $3 \cdot 7$ & $0 \cdot 4$ & $28 \cdot 9(2 \cdot 4-265 \cdot 1)$ \\
\hline Oceania $^{\mathrm{m}}$ & $0 \cdot 6$ & $0 \cdot 4$ & $0 \cdot 8(0 \cdot 0-43040 \cdot 2)$ n.s. \\
\hline Latin America and the Caribbean ${ }^{\mathrm{n}}$ & $4 \cdot 9$ & $0 \cdot 4$ & $20 \cdot 8(2 \cdot 0-211 \cdot 6)$ \\
\hline Eating chicken $v s$. not eating chicken (2) & $80 \cdot 0$ & $73 \cdot 7$ & $2 \cdot 0(1 \cdot 1-3 \cdot 5)$ \\
\hline Eating yoghurt $v s$. not eating yoghurt (4) & $62 \cdot 5$ & $88 \cdot 1$ & $0 \cdot 4(0 \cdot 2-0 \cdot 7)$ \\
\hline \multicolumn{4}{|l|}{ Eating vegetable salad vs. not eating vegetable salad (3) } \\
\hline When travelling within Europe & $27 \cdot 3$ & $27 \cdot 1$ & $1 \cdot 7(0 \cdot 9-3 \cdot 1)$ n.s. \\
\hline When travelling outside Europe & $17 \cdot 4$ & $2 \cdot 0$ & $6 \cdot 7(2 \cdot 1-40 \cdot 2)$ \\
\hline \multicolumn{4}{|l|}{$\begin{array}{l}\text { Drinking exclusively bottled water vs. drinking both } \\
\text { bottled and non-bottled water (3) }\end{array}$} \\
\hline When travelling within Europe, excluding Southern Europe & $6 \cdot 0$ & $8 \cdot 2$ & $1 \cdot 5(0 \cdot 6-3 \cdot 6)$ n.s. \\
\hline When travelling to Southern Europe and outside Europe & $33 \cdot 3$ & $7 \cdot 2$ & $2 \cdot 3(1 \cdot 6-5 \cdot 0)$ \\
\hline $\begin{array}{l}\text { Handling and/or eating raw or undercooked pork } v s \text {. not } \\
\text { handling and/or eating raw or undercooked pork (5) }\end{array}$ & $9 \cdot 8$ & $2 \cdot 3$ & $6 \cdot 2(1 \cdot 3-29 \cdot 3)$ \\
\hline Using proton-pump inhibitors $v s$. not using proton-pump inhibitors & $8 \cdot 5$ & $1 \cdot 6$ & $14 \cdot 6(3 \cdot 0-82 \cdot 0)$ \\
\hline $\begin{array}{l}\text { Having a chronic gastrointestinal disease } v s \text {. not having a chronic } \\
\text { gastrointestinal disease (5) }\end{array}$ & $6 \cdot 2$ & $3 \cdot 5$ & $2 \cdot 9(1 \cdot 7-4 \cdot 8)$ \\
\hline Working in healthcare $v s$. not working in healthcare (1) & $5 \cdot 7$ & $9 \cdot 0$ & $0 \cdot 4(0 \cdot 1-0 \cdot 9)$ \\
\hline
\end{tabular}

n.s., Not significant $(P>0 \cdot 05)$.

${ }^{\text {a }}$ Adjusted for age, sex, degree of urbanization, season and level of education.

${ }^{\mathrm{b}}$ Continuous variable, expressed as number of days stayed.

${ }^{\mathrm{c}}$ Adapted from the United Nations scheme of the composition of macro-geographical (continental) regions, geographical sub-regions, and selected economic and other groupings. (http://unstats.un.org/unsd/methods/m49/m49regin.htm\#europe).

${ }^{\mathrm{d}}$ Includes travellers returning from Germany, France, Belgium, Austria, Luxemburg, and Switzerland.

${ }^{\mathrm{e}}$ Includes travellers returning from the UK, Ireland, Denmark, Sweden, Norway, and Finland.

${ }^{\mathrm{f}}$ Includes travellers returning from the Czech Republic, Hungary, Poland, Slovakia, and Romania.

${ }^{\mathrm{g}}$ Includes travellers returning from Spain, Italy, Portugal, Greece, Croatia, and Malta.

${ }^{\mathrm{h}}$ Includes travellers returning from Morocco, Egypt, and Tunisia.

${ }^{\mathrm{i}}$ Includes travellers returning from Benin, Cameroon, Ethiopia, Ghana, Kenya, Mali, Nigeria, Rwanda, Tanzania, Botswana, Burkina Faso, South Africa and Namibia.

${ }^{\mathrm{j}}$ Includes travellers returning from Turkey, Jordan and Iraq.

${ }^{\mathrm{k}}$ Includes travellers returning from China, Indonesia, Malaysia, Singapore, Philippines, Thailand, and Vietnam.

${ }^{1}$ Includes travellers returning from India, Nepal, and Bangladesh.

${ }^{\mathrm{m}}$ Includes travellers returning from Australia and Fiji Islands.

${ }^{\mathrm{n}}$ Includes travellers returning from Bolivia, Ecuador, Peru, Venezuela, Chile, Costa Rica, Cuba, Dominican Republic, Haiti, Mexico, and Guatemala.

to conduct a stratified analysis by travel destination, it was possible to highlight some interactions between the exposures and the travel destinations.
Eight factors significantly associated with travelrelated campylobacteriosis were identified. Consistent with evidence that poultry is the main reservoir 
Table 4. Multivariable odds ratios (OR) and corresponding 95\% confidence intervals (CI) of risk factors for Campylobacter jejuni/coli infection acquired in The Netherlands caused by strains of most likely exotic origin

\begin{tabular}{|c|c|c|c|}
\hline Risk factor (\% of imputed missing values) & $\begin{array}{l}\% \text { cases } \\
\text { exposed } \\
(n=79)\end{array}$ & $\begin{array}{l}\% \text { controls } \\
\text { exposed } \\
(n=3119)\end{array}$ & $\begin{array}{l}\text { Multivariable } \\
\text { OR }(95 \% \mathrm{CI})^{\mathrm{a}}\end{array}$ \\
\hline $\begin{array}{l}\text { Contact with people with gastroenteritis outside } \\
\text { the household ( } 3 \text { ) }\end{array}$ & $17 \cdot 2$ & $10 \cdot 5$ & $2 \cdot 2(1 \cdot 9-4 \cdot 6)$ \\
\hline Recent use of proton-pump inhibitors & $15 \cdot 2$ & $2 \cdot 2$ & $9 \cdot 5(4 \cdot 4-20 \cdot 6)$ \\
\hline Eating yoghurt (2) & 71.9 & $86 \cdot 5$ & $0 \cdot 3(0 \cdot 2-0 \cdot 6)$ \\
\hline \multicolumn{4}{|l|}{ Not washing hands after toilet visit } \\
\hline April-December 2002 & $1 \cdot 3$ & $0 \cdot 6$ & $6 \cdot 8(0 \cdot 7-68 \cdot 1)$ n.s. \\
\hline January-April 2003 & $2 \cdot 5$ & $0 \cdot 1$ & $20 \cdot 8(1 \cdot 9-233 \cdot 4)$ \\
\hline \multicolumn{4}{|l|}{ Being a school-attending child } \\
\hline July-September 2002 and January-April 2003 & $6 \cdot 3$ & $6 \cdot 5$ & $1 \cdot 4(0 \cdot 5-3 \cdot 8)$ n.s. \\
\hline April-June 2002 & $5 \cdot 1$ & $2 \cdot 6$ & $3 \cdot 4(1 \cdot 0-11 \cdot 4)$ \\
\hline October-December 2002 & $7 \cdot 6$ & $2 \cdot 0$ & $4 \cdot 0(1 \cdot 4-11 \cdot 3)$ \\
\hline \multicolumn{4}{|l|}{ Swimming in a public swimming pool (1) } \\
\hline April-September 2002 and January-April 2003 & $10 \cdot 1$ & $11 \cdot 8$ & $1 \cdot 0(0 \cdot 4-2 \cdot 1)$ n.s. \\
\hline October-December 2003 & $7 \cdot 8$ & $2 \cdot 9$ & $3 \cdot 7(1 \cdot 3-11 \cdot 0)$ \\
\hline
\end{tabular}

n.s., Not significant $(P>0 \cdot 05)$.

${ }^{\mathrm{a}}$ Also adjusted for age, sex, degree of urbanization, season and level of education.

for campylobacters, most studies concerning risk factors for campylobacteriosis have identified an association with eating chicken $[6,7,16,36,37]$, suggesting that this factor is not exclusive for acquiring infection abroad. This also applies to consuming antacids and having chronic enteropathies $[6,7,16]$.

In The Netherlands, $<1 \%$ of domestically acquired Campylobacter infections have been attributed to pigs [16]. Moreover, eating pork has been associated with a reduced risk for $C$. coli [6] and chicken-borne C. jejunilcoli [16] infections. Accordingly, Dutch retail pork has rarely been found contaminated with campylobacters [38]. The positive association with handling and/or eating raw or undercooked pork we found therefore suggests that pigs may be an important reservoir (and pork an important exposure) of campylobacteriosis outside The Netherlands.

In contrast to previous findings indicating that eating vegetable salad is negatively associated with the occurrence of (domestic) campylobacteriosis [6, 16], we observed that this factor was associated with increased odds of acquiring campylobacteriosis when travelling outside Europe. In Europe, extensive sampling of raw vegetables, including ready-to-eat salads, has generally found no, or very few, campylobacters [38], suggesting that contamination of such items during irrigation, harvesting and processing is unlikely and that salads may occasionally become cross-contaminated during food preparation [36]. Conversely, exceptionally high Campylobacter isolation rates $(\sim 68 \%)$ in raw vegetables were reported from countries such as Malaysia [39], indicating that major problems can arise by consuming vegetables if hygiene practices are absent or break down.

Drinking bottled water exclusively was associated with increased odds of acquiring campylobacteriosis when travelling to high-risk destinations. In the UK, drinking bottled water has been found to be positively associated with campylobacteriosis [36], particularly C. coli infection [40], and ciprofloxacinresistant Campylobacter infection acquired abroad [5], suggesting that bottled water could, given the right circumstances, provide a vehicle for campylobacters [36]. In fact, bottled water, unlike tap water, is not usually treated and testing for Campylobacter is rarely undertaken $[36,40]$. Moreover, in the event of dual contamination of bottled water (campylobacters and organic matter), C. jejuni may survive for prolonged periods [41]. However, our association with bottled water was only significant when travelling to high-risk destinations, supporting the hypothesis that drinking bottled water acts as a proxy for local circumstances where there is a generally high risk for campylobacteriosis. Travellers are indeed usually advised to drink bottled water where there is any doubt about the local water quality. The use of bottled 
water may help in preventing infection but there may be circumstances where the risk is higher than that which can be prevented by drinking bottled water. Moreover, our questionnaire did not distinguish between sparkling and still bottled water and did not ask whether it was consumed with or without ice. Therefore, further investigation is needed to assess if the advice of drinking bottled water merits any refinement.

Proportionally more controls than cases reported consuming yoghurt. It is believed that probiotic bacteria in yoghurt may alter the intestinal microflora in a way that prevents infection [37], while people working in healthcare might be particularly aware of the health risks (and ways to avoid them) when travelling.

Putative determinants of exotically introduced domestic campylobacteriosis were suggestive of anthroponotic transmission, namely contact with gastroenteritis cases outside the household (thus less likely to share the same exposure); not washing hands after toilet visit; being a school-attending child (usually having high frequencies of contacts); and attending public swimming pools (as recreational water has been proposed as a vehicle for Campylobacter transmission [16, 37]). Except for the first factor, the others were unidentified in previous analyses where the same cases were not split according to their estimated exoticism [6, 16]. Moreover, we found significant interactions with season, which is in accordance with the seasonal nature of travelling, as also shown by the finding that $P r_{\mathrm{e}}$ varies seasonally. Periods most at risk were mainly those around popular holidays in The Netherlands, notably the autumn break in October, Christmas/New Year in December-January, and Easter in April-May. Moreover, people most at risk were school-attending children for which additional peaks in domestic campylobacteriosis have already been noted shortly after the end of school breaks, suggesting that these additional peaks are due to exposure to less common strains from less common foods consumed during the festivities and to the mixing of people that have not been in contact for a long time following on from the previous holidays [42]. It can therefore be hypothesized that travellers infected with strains possessing uncommon antigens might still be shedding them after returning home, most likely asymptomatically [14]. As there is unlikely to be a high prevalence of acquired protection against these exotic strains domestically, there is at least the potential for them to spread even through limited person-to-person transmission, with an average of $3 \cdot 2 \%$ domestic cases being estimated to be due to anthroponotic transmission of exotically introduced strains. In conclusion, this study sought to provide an outline of Campylobacter transboundary epidemiology that may apply to other countries as well. This ranges from the identification of $C$. jejunilcoli MLST genotypes causing infection in international travellers to the risk factors potentially responsible for acquiring infection with such strains upon travelling, as well as those potentially responsible for their secondary spread to domestic populations. Besides the identification of high-risk travel destinations and universal correlates of campylobacteriosis, such as eating chicken, using antacids and having chronic enteropathies, this study also identified eating vegetable salad outside Europe, drinking bottled water in high-risk destinations and handling and/or eating raw or undercooked pork as factors associated specifically with travel-related campylobacteriosis. Moreover, suggestive evidence was provided that international travellers are particularly prone to infection with Campylobacter MLST genotypes that are endemic in the different travel regions, and that these exotic strains may be carried by the returning travellers and be spread to domestic populations through limited person-to-person transmission. As travellers have dynamic interactions with people, places, and microbes during their journeys, it is conceivable that they can be victims, carriers, and eventually transmitters of such agents to new regions and populations. Our understanding of campylobacteriosis may therefore depend on increased insight into regional risk differences, high-risk exposures and Campylobacter behaviour in response to newly available susceptible populations and changing environments.

\section{ACKNOWLEDGEMENTS}

We are grateful to M. A. S. de Wit and Y. Doorduyn for their contribution in designing the study; to P. Mullner for help with access to data; and to the laboratory staff of the Central Veterinary Institute, especially E. Pothoven, for taking care of the strain collection. We also thank the participating Regional Public Health Laboratories for their contribution to the data collection, especially F. Vlaspolder, J. H. Sloos, J. Spaargaren, J. Peereboom, M. A. Schouten, R. W. Brimicombe, F.W. Sebens, H. Rothbarth, L. J. M. Sabbe, H. Mulder, D. Veenendaal, E. Ijzerman, J. H. T. Wagenvoort, J. H. van Zeijl, B. M. de Jongh, M. Tersmette, P. Voorn, A. M. Horrevorts, J. Buitenwerf, B. G. A. Hendrickx, M. Peeters and 
A. R. Jansz. Thanks are also extended to H.C. Boshuizen for help in multiple imputation method and to M. L. Callegari for help in data entry.

\section{DECLARATION OF INTEREST}

None.

\section{REFERENCES}

1. Belderok SM, et al. Incidence, risk factors and treatment of diarrhoea among Dutch travellers: reasons not to routinely prescribe antibiotics. BMC Infectious Diseases 2011; 11: 295.

2. Ekdahl K, Andersson Y. Regional risks and seasonality in travel-associated campylobacteriosis. BMC Infectious Diseases 2004; 4: 54.

3. Hakanen A, et al. Fluoroquinolone resistance in Campylobacter jejuni isolates in travelers returning to Finland: association of ciprofloxacin resistance to travel destination. Emerging Infectious Diseases 2003; 9: $267-70$.

4. Havelaar AH, et al. Disease burden of foodborne pathogens in the Netherlands, 2009. International Journal of Food Microbiology 2012; 156: 231-238.

5. Evans MR, et al. Risk factors for ciprofloxacin-resistant Campylobacter infection in Wales. Journal of Antimicrobial Chemotherapy 2009; 64: 424-427.

6. Doorduyn Y, et al. Risk factors for indigenous Campylobacter jejuni and Campylobacter coli infections in The Netherlands: a case-control study. Epidemiology and Infection 2010; 138: 1391-1404.

7. Tam CC, et al. Chicken consumption and use of acidsuppressing medications as risk factors for Campylobacter enteritis, England. Emerging Infectious Diseases 2009; 15: 1402-1408.

8. Hoge CW, et al. Epidemiology of diarrhea among expatriate residents living in a highly endemic environment. Journal of the American Medical Association 1996; 275: 533-538.

9. Gaudio PA, et al. Diarrhea among expatriate residents in Thailand: correlation between reduced Campylobacter prevalence and longer duration of stay. Journal of Travel Medicine 1996; 3: 77-79.

10. Cawthraw SA, et al. Antibodies, directed towards Campylobacter jejuni antigens, in sera from poultry abattoir workers. Clinical and Experimental Immunology 2000; 122: 55-60.

11. Miller G, et al. Does age acquired immunity confer selective protection to common serotypes of Campylobacter jejuni? BMC Infectious Diseases 2005; 5: 66.

12. Havelaar AH, et al. Estimating the true incidence of campylobacteriosis and salmonellosis in the European Union, 2009. Epidemiology and Infection 2013; 141: 293-302.

13. Smid JH, et al. Practicalities of using non-local or nonrecent multilocus sequence typing data for source attribution in space and time of human campylobacteriosis. PLOS ONE 2013; 8: e55029.
14. Havelaar AH, et al. Immunity to Campylobacter: its role in risk assessment and epidemiology. Critical Reviews in Microbiology 2009; 35: 1-22.

15. Arsenault $\mathbf{J}$, et al. Do patients with recurrent episodes of campylobacteriosis differ from those with a single disease event? BMC Public Health 2011; 11: 32.

16. Mughini Gras L, et al. Risk factors for campylobacteriosis of chicken, ruminant, and environmental origin: a combined case-control and source attribution analysis. PLOS ONE 2012; 7: e42599.

17. Ethelberg S, et al. Household outbreaks among cultureconfirmed cases of bacterial gastrointestinal disease. American Journal of Epidemiology 2004; 159: 406-412.

18. Rotariu O, et al. Putative household outbreaks of campylobacteriosis typically comprise single MLST genotypes. Epidemiology and Infection 2010; 138: 1744-1747.

19. Fermér C, Engvall EO. Specific PCR identification and differentiation of the thermophilic campylobacters, Campylobacter jejuni, C. coli, C. lari, and C. upsaliensis. Journal of Clinical Microbiology 1999; 37: 3370-3373.

20. Marshall SM, et al. Rapid identification of Campylobacter, Arcobacter, and Helicobacter isolates by PCR-restriction fragment length polymorphism analysis of the 16S rRNA gene. Journal of Clinical Microbiology 1999; 37: 4158-4160.

21. Doorduyn Y, et al. Risk factors for Salmonella Enteritidis and Typhimurium (DT104 and nonDT104) infections in The Netherlands: predominant roles for raw eggs in Enteritidis and sandboxes in Typhimurium infections. Epidemiology and Infection 2006; 134: 617-626.

22. Dingle KE, et al. Multilocus sequence typing system for Campylobacter jejuni. Journal of Clinical Microbiology 2001; 39: 14-23.

23. Hunter PR, Gaston MA. Numerical index of the discriminatory ability of typing systems: an application of Simpson's index of diversity. Journal of Clinical Microbiology 1988; 26: 2465-2466.

24. Wilson DJ, et al. Tracing the source of campylobacteriosis. PLoS Genetics 2008; 4: e1000203.

25. Strachan NJC, et al. Attribution of Campylobacter infections in northeast Scotland to specific sources by use of multilocus sequence typing. Journal of Infectious Diseases 2009; 199: 1205-1208.

26. Korczak BM, et al. Multiplex strategy for multilocus sequence typing, $f l a$ typing, and genetic determination of antimicrobial resistance of Campylobacter jejuni and Campylobacter coli isolates collected in Switzerland. Journal of Clinical Microbiology 2009; 47: 1996-2007.

27. Bessell PR, et al. Using sequence data to identify alternative routes and risk of infection: a case-study of campylobacter in Scotland. BMC Infectious Diseases 2012; 12: 80.

28. Mullner $\mathbf{P}$, et al. Molecular and spatial epidemiology of human campylobacteriosis: source association and genotype-related risk factors. Epidemiology and Infection 2010; 138: 1372-1383.

29. Strachan NJC, et al. Identifying the seasonal origins of human campylobacteriosis. Epidemiology and Infection 2013; 141: 1267-1275. 
30. McCarthy ND, et al. Molecular epidemiology of human Campylobacter jejuni shows association between seasonal and international patterns of disease. Epidemiology and Infection 2012; 140: 2247-55.

31. Mickan L, et al. Multilocus sequence typing of Campylobacter jejuni isolates from New South Wales, Australia. Journal of Applied Microbiology 2007; 102: 144-152.

32. McTavish SM, et al. Wide geographical distribution of internationally rare Campylobacter clones within New Zealand. Epidemiology and Infection 2008; 136: $1244-1252$.

33. Duim B, et al. Molecular evidence for dissemination of unique Campylobacter jejuni clones in Curaçao, Netherlands Antilles. Journal of Clinical Microbiology 2003; 41: 5593-5597.

34. Gupta S, Maiden MC. Exploring the evolution of diversity in pathogen populations. Trends in Microbiology 2001; 9: 181-185.

35. Kärenlampi R, et al. Longitudinal study of Finnish Campylobacter jejuni and C. coli isolates from humans, using multilocus sequence typing, including comparison with epidemiological data and isolates from poultry and cattle. Applied and Environmental Microbiology 2007; 73: $148-155$.

36. Evans MR, et al. Hazards of healthy living: bottled water and salad vegetables as risk factors for
Campylobacter infection. Emerging Infectious Diseases 2003; 9: 1219-1225.

37. Kapperud G, et al. Factors associated with increased and decreased risk of Campylobacter infection: a prospective case-control study in Norway. American Journal of Epidemiology 2003; 158: 234-242.

38. European Food Safety Authority and European Centre for Disease Prevention and Control. The European Union summary report on trends and sources of zoonoses, zoonotic agents and foodborne outbreaks in the European Union in 2010. EFSA Journal 2012; 10(6): 2765.

39. Chai LC, et al. Thermophilic Campylobacter spp. in salad vegetables in Malaysia. International Journal of Food Microbiology 2007; 117: 106-111.

40. Gillespie IA, et al. A case-case comparison of Campylobacter coli and Campylobacter jejuni infection: atool for generating hypotheses. Emerging Infectious Diseases 2002; 8: 937-942.

41. Tatchou-Nyamsi-König J-A, et al. Behaviour of Campylobacter jejuni in experimentally contaminated bottled natural mineral water. Journal of Applied Microbiology 2007; 103: 280-288.

42. van Pelt W, et al. Similarities and differences in seasonality of Campylobacter in broilers and humans, 1998-2006, the Netherlands. Zoonoses and Public Health 2007; 54: 51. 